\title{
Machine-to-machine communication: an overview of opportunities
}

\begin{abstract}
The envisioned capability of machine devices to autonomously communicate in the future Internet of Things (IoT) has brought considerable attention to machine-to-machine (M2M) communication in recent years. This paradigm has applications in homes, safety, transport, health, and industry. As an active focus of research, there are interesting open questions on several of its aspects, which we aim to capture in this paper. Accompanied by an attempted classification of existing surveys on $\mathrm{M} 2 \mathrm{M}$, we propose a followable exposition on the challenges and open research opportunities that embrace the diverse facets of M2M communication.
\end{abstract}

Keyword: Access problems; Applications; Challenges; Home; Mobile; Open issues; Standards; Survey; Taxonomy; Technologies; M2M; MAC 